\title{
NUMERICAL SIMULATION OF CHEMOTACTIC BACTERIA AGGREGATION VIA MIXED FINITE ELEMENTS
}

\author{
AMERICO MARROCCO ${ }^{1}$
}

\begin{abstract}
We start from a mathematical model which describes the collective motion of bacteria taking into account the underlying biochemistry. This model was first introduced by Keller-Segel [13]. A new formulation of the system of partial differential equations is obtained by the introduction of a new variable (this new variable is similar to the quasi-Fermi level in the framework of semiconductor modelling). This new system of P.D.E. is approximated via a mixed finite element technique. The solution algorithm is then described and finally we give some preliminary numerical results. Especially our method is well adapted to compute the concentration of bacteria.
\end{abstract}

Mathematics Subject Classification. 35Q, 65M, 92B, 92C.

\section{Problem formulation}

The equations for the collective motion of the bacteria can be derived (with no free parameters) from the underlying biochemistry. The basic equations for the bacterial density $\rho$ and the attractant concentration $c$ are $($ see $[1-3,13])$

where

$$
\begin{gathered}
\frac{\partial \rho}{\partial t}=D_{b} \nabla^{2} \rho-\nabla \cdot(k \rho \nabla c)+a \rho, \\
\frac{\partial c}{\partial t}=D_{c} \nabla^{2} c+\alpha \rho,
\end{gathered}
$$

$$
\begin{aligned}
& D_{b} \text { is the bacterial diffusion constant, } \\
& k \text { is the chemotactic coefficient (or chemotactic sensitivity), } \\
& a \text { is the rate of bacterial division, } \\
& \alpha \text { is the rate of attractant production, } \\
& D_{c} \text { is the chemical diffusion constant. }
\end{aligned}
$$

The terms in equation (1) include the diffusion of bacteria, chemotactic drift and division of bacteria. Equation (2) expresses the diffusion and production of attractant. In this model, the production of attractants is taken proportional to the bacterial density, as a first approach. But other models, certainly more realistic exists, see for example the model given by equation (7).

Keywords and phrases. Biophysics, chemotaxis, numerical simulation, mixed finite element.

1 Inria, Domaine de Voluceau, Rocquencourt, BP 105, 78153 Le Chesnay, France. e-mail: Americo.Marrocco@requin.inria.fr 
In [1] for example, typical numerical values are given for the various parameters,

$$
\begin{aligned}
D_{b} & =7.10^{-6} \mathrm{~cm}^{2} / \mathrm{s}, D_{c}=10^{-5} \mathrm{~cm}^{2} / \mathrm{s}, \\
k & =10^{-16} \mathrm{~cm}^{5} / \mathrm{s}, \quad \alpha=10^{3} / \mathrm{s} / \text { bacteria. }
\end{aligned}
$$

Taking a bacterial density $\rho=10^{6} / \mathrm{cm}^{3}$ the length scale is about 260 microns and the time scale $100 \mathrm{~s}$.

In some semi-solid media (like agar), the diffusion of bacteria is much slower than attactant diffusion which motivate to drop the term with time derivative in equation (2). This limit case is also convenient for asymptotic behaviour when considering other media. Notice that for other applications, the diffusion of attractants is neglected, this is the case for angiogenesis, see [4] and the references therein.

In addition, cells divide much more slowly than the dynamics take place (the time scale for cell division is about 2 hours) so that we can take $a=0$ in (1) for an entry level model. The model used for the numerical simulations is then the following

$$
\begin{gathered}
\frac{\partial \rho}{\partial t}=D_{b} \nabla^{2} \rho-\nabla \cdot(k \rho \nabla c), \\
0=D_{c} \nabla^{2} c+\alpha \rho,
\end{gathered}
$$

which we re-write (for convenience)

$$
\begin{gathered}
-\operatorname{div}\left(\mathrm{D}_{\mathrm{c}} \operatorname{grad} \mathrm{c}\right)-\alpha \rho=0, \\
\frac{\partial \rho}{\partial t}-\operatorname{div}\left(\mathrm{D}_{\mathrm{b}} \operatorname{grad} \rho-\mathrm{k} \rho \operatorname{grad} \mathrm{c}\right)=0 .
\end{gathered}
$$

This last formulation ((5)-(6)), strongly looks like a classical model of transient drift-diffusion of electrons in semiconductor media (nevertheless the term wich corresponds here to the attractant production is with an opposite sign). The attractant concentration $c$ corresponds to the electrostatic potential $(\varphi)$, the bacterial density $\rho$ to the electron density $(n)$, the chemotactic coefficient $k$ to the electron mobility $\left(\mu_{n}\right)$. As in semiconductor modelling framework, a new formulation of system ((5)-(6)) will be obtained by introduction of a variable which is similar to the electron quasi-Fermi level and denoted by $\varphi_{n}$.

Note that we can find in the paper [1], one other model for equation (4)

$$
0=D_{c} \nabla_{c}^{2}+\alpha \rho e^{-\rho / \rho^{*}},
$$

which can be used when modelling the following fact: when the bacterial density becomes too high, the bacteria consume all the food sources (succinate and oxygen) in their local environment and cease producing the attractant aspartate. The parameter $\rho^{*}$ is the cutoff bacterial density.

One of the problem that has attracted biologists and mathematicians is to understand pattern formation in such models. The most noticeable, and complex also is the aggregation of bacteria $[1,2,8,9,12]$. In this paper we investigate this pattern based on a mixed finite element method. We begin with another formulation based on quasi-Fermi levels, then we describe the finite element algorithm and we present in a fourth section some numerical results.

\section{New Formulation of the "Chemotaxis Model"}

First of all we can introduce a scaling on the $c$ variable (attractant concentration) via the parameter $q$ ( $q$ has no special physical meaning and is only introduced for numerical convenience),

$$
c=\frac{\widetilde{c}}{q} .
$$

The system ((5)-(6)), becomes

$$
-\operatorname{div}\left(D_{c} \operatorname{grad} \tilde{c}\right)-q \alpha \rho=0,
$$




$$
\frac{\partial \rho}{\partial t}-\operatorname{div}\left[\mathrm{D}_{\mathrm{b}} \operatorname{grad} \rho-\frac{\mathrm{k}}{\mathrm{q}} \rho \operatorname{grad} \tilde{\mathrm{c}}\right]=0,
$$

$\left(+\frac{\partial \tilde{c}}{\partial t}\right.$ could be added to the left hand side of (9) if the transient equation for the attractant diffusion was considered). We introduce a new variable $\varphi_{n}$ by setting

$$
\rho=\rho\left(\tilde{c}, \varphi_{n}\right)=\rho_{0} e^{\frac{\tilde{c}+\varphi_{n}}{2}},
$$

where $\rho_{0}$ is a constant (which corresponds to a given bacterial density, the variable $\tilde{c}$ and $\varphi_{n}$ (or their sum) are then equal to zero), and $Z=\frac{q D_{b}}{k}$.

This class of variables is useful because we expect high values of $\rho$ (collapse) and the potential $\varphi_{n}$ is a more reasonable quantity. The system of equations can now be written as

$$
\begin{gathered}
-\operatorname{div}\left(\mathrm{D}_{\mathrm{c}} \operatorname{grad} \tilde{\mathrm{c}}\right)-\tilde{\alpha} \rho\left(\tilde{\mathrm{c}}, \varphi_{\mathrm{n}}\right)=0, \\
\frac{\partial \rho\left(\tilde{c}, \varphi_{n}\right)}{\partial t}-\operatorname{div}\left(\tilde{\mathrm{k}} \rho\left(\tilde{\mathrm{c}}, \varphi_{\mathrm{n}}\right) \operatorname{grad} \varphi_{\mathrm{n}}\right)=0,
\end{gathered}
$$

where

$$
\tilde{k}=k / q, \tilde{\alpha}=\alpha q .
$$

The system of equation ((12)-(13)) where the unknowns are $\tilde{c}$ and $\varphi_{n}$ will be used for the numerical approximation. This system has to be completed with appropriate boundary condition on $\tilde{c}$ and $\varphi_{n}$ and initial condition for the bacterial density $\rho$.

Equation (12) is similar to the Poisson equation and equation (13) to the electron continuity equation in the semiconductor framework.

\section{Mixed finite Element approximation And SOlution Algorithm}

\subsection{Numerical approximation (M.F.E.)}

Following $[5,6,10,11]$, and assuming that Dirichlet boundary condition on a part of the boundary $\left(\Gamma_{d}\right)$ - of the computational domain $\Omega$ - and Neumann boundary condition on the complementary boundary part $\left(\Gamma_{n}\right)$ hold for the variables $\tilde{c}$ and $\varphi_{n}$, we introduce the flux variables

$$
\vec{V}=D_{c} \operatorname{grad} \tilde{c}, \quad \vec{J}_{\rho}=\tilde{k} \rho\left(\tilde{c}, \varphi_{n}\right) \operatorname{grad} \varphi_{n}
$$

and an equivalent formulation of the system ((12)-(13)). The part of the boundary on which a Neumann condition holds for the $\tilde{c}$ variable is not necessarly the same as the one for the $\varphi_{n}$ variable. For this, an additional index is used in the following equations:

$$
\left\{\begin{aligned}
-\operatorname{div} \vec{V}-\tilde{\alpha} \rho\left(\tilde{c}, \varphi_{n}\right)=0 & \text { in } \Omega, \\
\vec{V}=D_{c} \operatorname{grad} \tilde{c} & \text { in } \Omega, \\
\vec{V} \cdot \vec{n}=D_{c} \cdot f_{1 n} & \text { on } \Gamma_{n 1}, \\
\tilde{c}=f_{1 d} & \text { on } \Gamma_{d 1},
\end{aligned}\right.
$$




$$
\left\{\begin{aligned}
\frac{\partial \rho\left(\tilde{c}, \varphi_{n}\right)}{\partial t}-\operatorname{div} \vec{J}_{\rho}=0 & \text { in } \Omega, \\
\vec{J}_{\rho}=\tilde{k} \rho\left(\tilde{c}, \varphi_{n}\right) \operatorname{grad} \varphi_{n} & \text { in } \Omega, \\
\vec{J}_{\rho} \cdot \vec{n}=\tilde{k} \rho f_{2 n} & \text { on } \Gamma_{n 2}, \\
\varphi_{n}=f_{2 n} & \text { on } \Gamma_{d 2},
\end{aligned}\right.
$$

$f_{1 d}$ and $f_{1 n}$ are given boundary conditions on $\tilde{c}$ (respectively of Dirichlet and Neumann type), and $f_{2 d}$ and $f_{2 n}$ similarly for $\varphi_{n}$.

The system we have to consider is now made up of four equations ((15)-(16)-(19)-(20)) with the four unknowns $\left(\tilde{c}, \vec{V}, \varphi_{n}, \vec{J}_{\rho}\right)$.

We consider now the following Sobolev spaces

$$
\begin{aligned}
& H(\operatorname{div})=\left\{w \mid w \in\left(L^{2}(\Omega)\right)^{2}, \quad \operatorname{div} w \in L^{2}(\Omega)\right\}, \\
& V_{0, i}=\left\{w \mid w \in H(\operatorname{div}), \quad w \cdot n=0 \text { on } \Gamma_{n, i}\right\} .
\end{aligned}
$$

Then, for sufficiently smooth data we can obtain the following equivalent dual mixed variational formulation of the original problem.

Find $\vec{V} \in H(\operatorname{div}), \quad \tilde{c} \in L^{2}(\Omega)$, $\vec{J}_{\rho} \in H(\operatorname{div}), \quad \varphi_{n} \in L^{2}(\Omega)$ such that

$$
\begin{gathered}
-\int_{\Omega} \operatorname{div} \vec{V} \cdot v_{1} \mathrm{~d} x-\int_{\Omega} \tilde{\alpha} \rho\left(\tilde{c}, \varphi_{n}\right) \cdot v_{1} \mathrm{~d} x=0 \quad \forall v_{1} \in L^{2}(\Omega), \\
\int_{\Omega}\left[D_{c}\right]^{-1} \vec{V} \cdot \vec{w}_{1} \mathrm{~d} x+\int_{\Omega} \tilde{c} \cdot \operatorname{div} \vec{w}_{1} \mathrm{~d} x-\int_{\Gamma_{d 1}} f_{1 d} \cdot \vec{w}_{1} \cdot \vec{n} \mathrm{~d} \Gamma=0 \quad \forall \vec{w}_{1} \in V_{0,1}, \\
\vec{V} \cdot \vec{n}=D_{c} f_{1 n} \text { on } \Gamma_{n, 1}, \\
\int_{\Omega} \frac{\partial \rho\left(\tilde{c}, \varphi_{n}\right)}{\partial t} \cdot v_{2} \mathrm{~d} x-\int_{\Omega} \operatorname{div} \vec{J}_{\rho} \cdot v_{2} \mathrm{~d} x=0 \quad \forall v_{2} \in L^{2}(\Omega), \\
\int_{\Omega}\left[\tilde{k} \rho\left(\tilde{c}, \varphi_{n}\right)\right]^{-1} \vec{J}_{\rho} \cdot \vec{w}_{2} \mathrm{~d} x+\int_{\Omega} \varphi_{n} \cdot \operatorname{div} \vec{w}_{2} \mathrm{~d} x-\int_{\Gamma_{d 2}} f_{2 d} \cdot \vec{w}_{2} \cdot \vec{n} \mathrm{~d} \Gamma=0 \quad \forall \vec{w}_{2} \in V_{0,2}, \\
\vec{J}_{\rho} \cdot \vec{n}=\tilde{k} \rho\left(\tilde{c}, \varphi_{n}\right) \cdot f_{2 n} \text { on } \Gamma_{n, 2} .
\end{gathered}
$$

For the numerical applications (as often it happens) we will have $f_{1 n} \equiv f_{2 n} \equiv 0$ so that we look for $\vec{V}$ and $\vec{J}_{\rho}$ in $V_{0,1}$ and $V_{0,2}$ respectively.

As in $[6,10,14,15]$ the previous formulation $((25)-(27))$ and $((28)-(30))$ allows us to realize easily a discrete approximation via mixed finite elements.

We retain the lowest order M.F.E. of Raviart-Thomas $\left(R_{0}^{T}\right)$ for implementation. After a triangulation $\mathcal{T}_{h}$ of the computational domain $\Omega \subset \mathbb{R}$, we define the following finite dimensional spaces

$$
\begin{gathered}
L_{h}=\left\{v_{h} \in L^{2}(\Omega)\left|\forall K \in \mathcal{T}_{h}, \quad v_{h}\right|_{K}=\text { constant }\right\}, \\
V_{h}=\left\{w_{h} \in H(\operatorname{div})\left|\forall K \in \mathcal{T}_{h}, \quad w_{h}(x, y)\right|_{K}=\left.\right|_{\beta_{K}} ^{\alpha_{K}}+\left.\gamma_{K}\right|_{y} ^{x}\right\} .
\end{gathered}
$$


We assume also that $\Gamma_{n, i}(i=1,2)$ is obtained by union of edges of triangles which belong to the mesh $\mathcal{T}_{h}$ and that

$$
V_{0, i, h}=V_{0, i} \cap V_{h} .
$$

Then the discrete formulation of the problem follows directly from $((25)-(30))$ by replacing $L^{2}(\Omega)$ by $L_{h}$ and $V_{0, i}$ by $V_{0, i, h}$.

\subsection{Solution algorithm}

Refering again to the publications related to semiconductor area $[5,6,10,14,15]$, various algorithms are described for a "stationary" version of system $((25)-(30))$ (i.e. without $\frac{\partial \rho}{\partial t}$ term !). Let us recall here the major characteristics of these numerical schemes:

- association to each (stationary) equation (primal variable) of the system of an "artificial" transient equation;

- semi-implicit (Peaceman Rachford, Douglas Rachford) or implicit (Backward-Euler) time discretization schemes for the "artificial" evolution equations. Use of local time steps;

- relaxation on the equations at each time step;

- for each time step, and for each equation of the system, solution of the nonlinear problem by NewtonRaphson technique.

The fact that we associate an "evolution" equation and the use of implicit or semi-implicit schemes is an other way to consider augmented Lagrangian techniques for the solution of saddle point problems [7] arising from the mixed formulation. Local time steps are closely connected with the penalty functions in the framework of Augmented Lagrangian techniques.

Special features of such an approach are relative simplicity and ease of implementation of model extensions. It is easy to add one equation to the system without need of deep changes in the algorithm architecture. Equations are handled successively in the algorithmic process. This numerical scheme, specially when using backward Euler for time discretization, has appeared to be rather efficient for semiconductor device (drift diffusion model and standard applied potentials). One can think reasonably that if the coupling between the equations of the system is not "too strong" then the relaxation on the equations do not penalize strongly the (speed of) convergence of the iterates towards the stationary solution of the whole system. This is certainly what happens in the static drift diffusion model for regimes not so far from the equilibrium state.

Nevertheless this solution technique failed when applied to energy-transport models for semiconductors [9,12]. One equation (relative to electron temperature) has been added to the drift diffusion model and the constitutive relation for the current density has been slightly modified. The previous numerical scheme works correctly only for solutions very close to the equilibrium state. In order to recover the algorithmic behaviour, for typical load range, similar to the behaviour, obtained when using the drift diffusion model, one has to consider simultaneously (couple) the equations relative to the electron current continuity and the energy [9]. The implementation of such numerical scheme in a little bit more complicated but seems a necessity to insure the convergence of the iterative procedure.

In past few years we are also interested by the numerical simulation of the transient behaviour (physical transient) of a semiconductor device governed by the drift diffusion model (numerical simulation of the switching of a diode). The model is very close to the "chemotaxis model".

After the semi-discretization in time, using fully implicit scheme, we have to deal with a sequence of "quasistatic" problems which are similar to the static drift diffusion problem himself, but generally easier to solve because the nonlinearities involved are of lower amplitude. The degree of nonlinearity may be controlled in practice by the physical time step. For "very small time step" the operator governing the transient tends to the identity operator (linear operator) and for sufficiently large time steps, the operator corresponds to the static drift diffusion (fully non linear operator).

The first attempt for the numerical simulation of the transient behaviour was to apply the most efficient scheme developped for the static drift diffusion model at each "quasi static" problem obtained via the semidiscretization in time of the transient model. As the time step (physical time step) becomes smaller and smaller the convergence of the iterates becomes more and more difficult to obtain and solution of quasi-static problems 
is not reached. The whole algorithm failed to converge. The only plausible explanation to this algorithm behaviour is that, as the time step (physical time step) becomes smaller, the coupling between the equations (Poisson equation and continuity equations) becomes stronger, and the relaxation strategy (decoupling) used in the numerical algorithm is no more adapted. A more implicit scheme has to be used for the solution of the quasi-static problems. The fully implicit scheme (on the system and not on each equation of the system) has been implemented and convergence was restored.

We describe now an adaptation of this numerical scheme as simply as possible.

A) Semi-discretization in time (physical time), by a fully implicit scheme (Backward-Euler).

The solution of the problem represents an approximation of the transient solution at different times $T_{n+1}=$ $T_{n}+\delta t(n=0,1,2,3, \ldots)$; the time step $\delta t$ may be adapted if needed. The formulation of the sequence of quasi-static problems is the following:

Find $\left(\tilde{c}^{n+1}, \vec{V}^{n+1}, \varphi_{n}^{n+1}, \vec{J}_{\rho}^{n+1}\right)$ solution of

$$
\begin{gathered}
-\operatorname{div} \vec{V}^{n+1}-\tilde{\alpha} \rho\left(\tilde{c}^{n+1}, \varphi_{n}^{n+1}\right)=0, \\
\int_{\Omega}\left[D_{c}\right]^{-1} \vec{V}^{n+1} \cdot w_{1} \mathrm{~d} x+\int_{\Omega} c^{n+1} \operatorname{div} w_{1} \mathrm{~d} x-\int_{\Gamma_{d 1}} f_{1 d} w_{1} \cdot n \mathrm{~d} \Gamma=0, \quad \forall w_{1} \in V_{0,1, h}, \\
-\operatorname{div} \vec{J}_{\rho}+\frac{1}{\delta t}\left[\rho\left(\tilde{c}^{n+1}, \varphi_{n}^{n+1}\right)-\rho\left(\tilde{c}^{n}, \varphi_{n}^{n}\right)\right]=0, \\
\int_{\Omega}\left[\tilde{k} \rho\left(\tilde{c}^{n+1}, \varphi_{n}^{n+1}\right)\right]^{-1} \vec{J}_{\rho} \cdot w_{2} \mathrm{~d} x+\int_{\Omega} \varphi_{n}^{n+1} \cdot \operatorname{div} w_{2} \mathrm{~d} x-\int_{\Gamma_{d 2}} f_{2 d} w_{2} \cdot n \mathrm{~d} \Gamma=0, \quad \forall w_{2} \in V_{0,2, h} .
\end{gathered}
$$

B) Each quasi-static problem (index $n$ ) is solved via artificial transient [6,15]. The index of time discretization is here $k$ (Backward Euler scheme on the system of equation)

$$
\begin{gathered}
\tilde{c}^{k+1}-\tilde{c}^{k}-\Delta t_{1}(x) \operatorname{div} \vec{V}^{k+1}-\Delta t_{1}(x) \cdot \tilde{\alpha} \rho\left(\tilde{c}^{k+1}, \varphi_{n}^{k+1}\right)=0, \\
\int_{\Omega}\left[D_{0}\right]^{-1} \vec{V}^{k+1} \cdot w_{1} \mathrm{~d} x+\int_{\Omega} c^{k+1} \operatorname{div} w_{1} \mathrm{~d} x-\int_{\Gamma_{d 1}} f_{1 d} w_{1} \cdot n \mathrm{~d} \Gamma=0, \quad \forall w_{1} \in V_{0,1, h}, \\
\varphi_{n}^{k+1}-\varphi_{n}^{k}-\Delta t_{2}(x) \operatorname{div} \vec{J}_{\rho}^{k+1}+\Delta t_{2}(x) \cdot \frac{1}{\partial t}\left[\rho\left(\tilde{c}^{k+1}, \varphi_{n}^{k+1}\right)-\rho^{n}\right]=0, \\
\int_{\Omega}\left[\tilde{k} \rho\left(\tilde{c}^{k+1}, \varphi_{n}^{k+1}\right) \vec{J}_{\rho}^{k+1} \cdot w_{2} \mathrm{~d} x+\int_{\Omega} \varphi_{n}^{k+1} \cdot \operatorname{div} w_{2} \mathrm{~d} x-\int_{\Gamma_{d 2}} f_{2 d} w_{2} \cdot n \mathrm{~d} \Gamma=0, \quad \forall w_{2} \in V_{0,2, h} .\right.
\end{gathered}
$$

In (40) $\rho^{n}$ is given $\left(\rho^{n}=\rho\left(\tilde{c}^{n}, \varphi_{n}^{n}\right)\right)$. In this process indexed with $k$, we can take $\tilde{c}^{0}=\tilde{c}^{n}$ and $\varphi_{n}^{0}=\varphi_{n}^{n}$. The unknowns are here $\left(\tilde{c}^{k+1}, \vec{V}^{k+1}, \varphi_{n}^{k+1}, \vec{J}_{\rho}^{k+1}\right)$. When the convergence with respect to $k$ is obtained, then the current solution gives $\left(\tilde{c}^{n+1}, \vec{V}^{n+1}, \varphi_{n}^{n+1}, \vec{J}_{\rho}^{n+1}\right)$.

As we use the lowest order Raviart-Thomas element, the equations (38) and (40) lead to scalar relation on each elements $T$ of the triangulation $\mathcal{T}_{h}$ (it is natural in this approximation to take the "local time steps" $\Delta t_{1}$ and $\Delta t_{2}$ constant on each element $\left.T\right)$.

C) At each step $k$, we have a non linear problem to solve. We use Newton-Raphson technique, the index of iteration is now denoted by $\ell$. We set:

$$
\vec{U}=\left(\begin{array}{c}
\tilde{c} \\
\vec{V} \\
\varphi_{n} \\
\vec{J}_{\rho}
\end{array}\right)=\left(\begin{array}{c}
U_{1} \\
U_{2} \\
U_{3} \\
U_{4}
\end{array}\right)
$$


Equations (38) and (39) can formally be written as

$$
\begin{gathered}
E_{1}\left(\tilde{c}, \vec{V}, \varphi_{n}, \cdot\right)=E_{1}\left(U_{1}, U_{2}, U_{3}, U_{4}\right)=E_{1}(\vec{U})=0, \\
E_{2}(\tilde{c}, \vec{V}, \cdot, \cdot)=E_{2}\left(U_{1}, U_{2}, U_{3}, U_{4}\right)=E_{2}(\vec{U})=0
\end{gathered}
$$

and so on, and finally the system $((38)-(41))$ may be synthetized by

$$
\vec{E}(\vec{U})=0
$$

The Newton-Raphson rule applied to (43) gives

$$
\left[\frac{\partial E_{i}}{\partial U_{j}}\right]_{\ell} \delta \vec{U}^{\ell}=-\vec{E}(\vec{U})^{\ell}, \quad \delta \vec{U}^{\ell}=\vec{U}^{\ell+1}-\vec{U}^{\ell},
$$

or equivalently

$$
\vec{U}^{\ell+1}=\vec{U}^{\ell}-\left[\frac{\partial E_{i}}{\partial U_{j}}\right]_{\ell}^{-1} \vec{E}(\vec{U})^{\ell}
$$

where $\left[\frac{\partial E_{i}}{\partial U_{j}}\right]_{\ell}$ is the jacobian matrix of the application $\vec{E}$ evaluated at step $\ell$.

The Newton-Raphson technique leads to a sequence of linear systems (indexed by $\ell$ ). In our context of approximation (M.F.E.- $R T_{0}$ ) the size of each linear system is approximatively

$$
2 \times(N T+N A)
$$

where $N T$ is the number of triangles of the triangulation $\mathcal{T}_{h}$, and $N A$ is the number of edges of the mesh.

On can reduce the size of linear system by eliminating the primal variables (i.e., $\tilde{c}$ and $\left.\varphi_{n}\right)$.

As a matter of fact, if we consider the first and third rows of relation (44), we can express explicitely the primal variables $\left(\tilde{c}^{\ell+1}, \varphi_{n}^{\ell+1}\right)$ as functions of the dual variables $\vec{V}^{\ell+1}$ and $\vec{J}_{\rho}^{\ell+1}$ by

$$
[A]\left[\begin{array}{c}
\tilde{c}^{\ell+1}-\tilde{c}^{\ell} \\
\varphi_{n}^{\ell+1}-\varphi_{n}^{\ell}
\end{array}\right]=\left[\begin{array}{c}
\Delta t_{1} \cdot \operatorname{div} \delta \vec{V}^{\ell} \\
\Delta t_{2} \operatorname{div} \delta \vec{J}_{\rho}^{\ell}
\end{array}\right]-\left[\begin{array}{c}
E_{1}\left(\vec{U}^{\ell}\right) \\
E_{3}\left(\vec{U}^{\ell}\right)
\end{array}\right] .
$$

The matrix $[A]$ is given by

$$
[A]=\left[\begin{array}{cc}
1-\left.\Delta t_{1} \tilde{\alpha} \rho_{\tilde{c}}^{\prime}\right|_{\ell} & -\left.\Delta t_{1} \tilde{\alpha} \rho_{\varphi_{n}}^{\prime}\right|_{\ell} \\
\left.\frac{\Delta t_{2}}{\delta t} \rho_{\tilde{c}}^{\prime}\right|_{\ell} & 1+\left.\frac{\Delta t_{2}}{\delta t} \rho_{\varphi_{n}}^{\prime}\right|_{\ell}
\end{array}\right] .
$$

By the definition of $\rho$ in (11), the derivatives of $\rho$ with respect to $\tilde{c}$ and $\varphi_{n}$ are the same and the determinant of $A$ can be evaluated as

$$
\operatorname{det} A=1+\rho_{\tilde{c}}^{\prime}\left(\frac{\Delta t_{2}}{\delta t}-\Delta t_{1} \cdot \tilde{\alpha}\right) .
$$

We can eventually act on the physical time step $\delta t$ or the local time steps $\Delta t_{1}$ and $\Delta t_{2}$ in order to keep $\operatorname{det} A \neq 0$ or even $>0$.

Let us denote by $B$ the inverse of matrix $A$

$$
B=A^{-1}
$$


Then the following relations are obtained for $\tilde{c}^{\ell+1}$ and $\varphi_{n}^{\ell+1}$

$$
\begin{aligned}
\tilde{c}^{\ell+1}-\tilde{c}^{\ell}=\delta \tilde{c}^{\ell}= & B_{11} \Delta t_{1} \operatorname{div} \vec{V}^{\ell+1}-B_{11}\left[\tilde{c}^{\ell}-\tilde{c}^{k}-\Delta t_{1} \tilde{\alpha} \rho^{\ell}\right] \\
& \left.+B_{12} \Delta t_{2} \operatorname{div} \vec{J}_{\rho}^{\ell+1}-B_{12}\left[\varphi_{n}^{\ell}-\varphi_{n}^{k}+\frac{\Delta t_{2}}{\delta t}\left(\rho^{\ell}-\rho^{n}\right)\right]\right\}, \\
\varphi_{n}^{\ell+1}-\varphi_{n}^{\ell}=\delta \varphi_{n}^{\ell} & =B_{21} \Delta t_{1} \operatorname{div} \vec{V}^{\ell+1}-B_{21}\left[\tilde{c}^{\ell}-\tilde{c}^{k}-\Delta t_{1} \tilde{\alpha} \rho^{\ell}\right] \\
& \left.+B_{22} \Delta t_{2} \operatorname{div} \vec{J}_{\rho}^{\ell+1}-B_{22}\left[\varphi_{n}^{\ell}-\varphi_{n}^{k}+\frac{\Delta t_{2}}{\delta t}\left(\rho^{\ell}-\rho^{n}\right)\right]\right\} .
\end{aligned}
$$

These relations (50) and (51) are then used within the second and fourth rows of (45). Finally the linear system we have to solve is relative to the unknowns $\vec{V}^{\ell+1}$ and $\vec{J}_{\rho}^{\ell+1}$ only (i.e. of maximum size 2.NA). When the convergence with respect to $\ell$ is reached then the solution at step $k$ of the whole process is obtained.

\section{NUMERICAL APPLiCATIONS}

We take for $\Omega$ the rectangular domain $L \times \ell$ (see Fig. 1) and we suppose that at $T=0$ we have a uniform distribution of bacteria with density $\rho_{0}$.
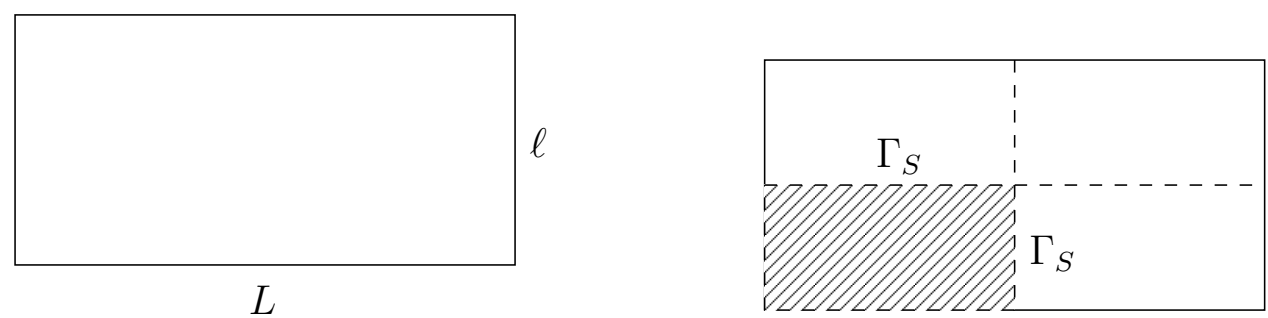

FiguRE 1. Simulation domain and reduction by symmetry.

From the definition of $\rho$ in (11) it is natural to take at initial time

$$
\tilde{c}+\varphi_{n}=0 .
$$

By analogy with the drift diffusion model for semiconductor the variable $\tilde{c}$ is similar to the electrostatic potential which is defined up to an additive constant. We can fix this constant by taking the value 0 . On the other hand we want to ensure in the following numerical approximation, the mass conservation (or volume conservation) for the bacteria. From equation (13) this will be the case if we take the following boundary condition on $\varphi_{n}$

In short, we can take at initial time

$$
\frac{\partial \varphi_{n}}{\partial n}=0 \quad \text { on } \quad \Gamma=\partial \Omega
$$

and the boundary condition

$$
\rho=\rho_{0} \quad \text { and } \quad \tilde{c}=\varphi_{n} \equiv 0,
$$

$$
\left.\tilde{c}\right|_{\Gamma}=0,\left.\quad \frac{\partial \varphi_{n}}{\partial n}\right|_{\Gamma}=0 .
$$

Of course, by reason of symmetry we can consider only a quarter of the simulation domain $\Omega$, namely $(L / 2, \ell / 2)$, then we have to take the following boundary conditions on the boundaries of symmetry $\Gamma_{S}$

$$
\frac{\partial \tilde{c}}{\partial n}=0, \quad \text { and } \quad \frac{\partial \varphi_{n}}{\partial n}=0 \quad \text { on } \quad \Gamma_{S} .
$$


The bacterial density is also assumed to be larger than a prescribed minimum value $\rho_{\min }$ (projection). We choose in our simulations

$$
\rho_{\min }=10^{-10} / \mathrm{cm}^{3} .
$$

We present now a typical result obtained for the numerical simulation of the chemotaxis model on a rectangular domain $\Omega=(1.5 \mathrm{~cm} \times 0.5 \mathrm{~cm})$. As mentioned above only a quarter of $\Omega$ is meshed and used for effective computations. We can see in Figure 2 the triangular mesh used for the numerical simulations.

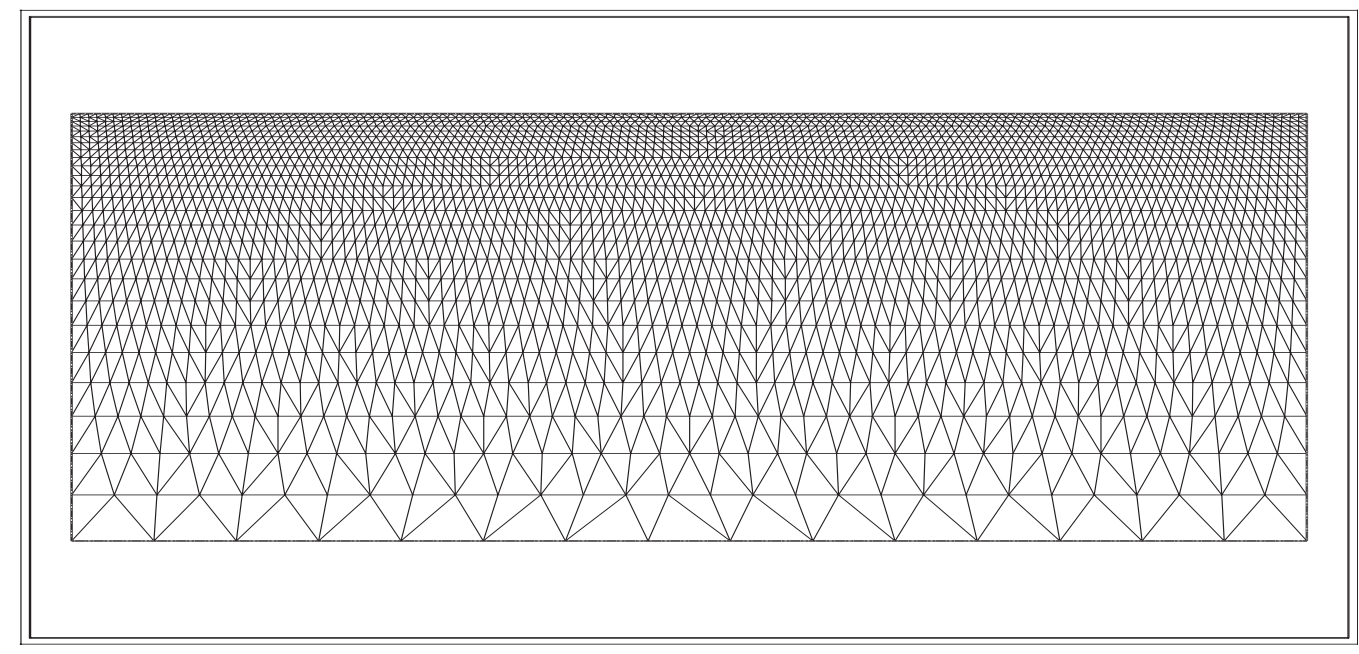

Figure 2. Meshes. The mesh contains 5433 triangles.

Two computations have been carried out:

a) the first with a production of attractants proportional to the bacterial density (model 1 given by equation (4);

b) the second with a limited production of attractants (model 2 given by eq. (7), with threshold value $\left.\rho^{*}=500 \rho_{o}\right)$.

The initial uniform distribution of bacteria is taken $\rho_{0}=10^{6} / \mathrm{cm}^{3}$. In (8) the scaling factor $q$ is taken as $10^{-12}$ in order to obtain "relative small" values for $\tilde{c}$ and $\varphi_{n}$. The initial constant value $\rho_{0}$ is marked on the figures.

The total amount of bacteria remain constant along the evolution process:

$$
\int_{\Omega} \rho \mathrm{d} x=C^{t e}=\int_{\Omega} \rho_{0} \mathrm{~d} x=\rho_{0} \times \operatorname{Area}(\Omega) .
$$

The coordinates $\left(x_{1}, x_{2}\right)$ are given in $\mu \mathrm{m}$ on the figures and a log scale is used for representation of the density $\rho$ (range from $10^{-10}$ to $10^{+10}\left(\mathrm{~cm}^{-3}\right)$ ).

Some other numerical simulations can be found in [16].

\subsection{Numerical results with attractant production proportional to the bacteria density}

Figure 3 present the bacteria density distribution after $100 \mathrm{~s}$. A peak of concentration begins to appear at the point $\approx(0.273,0.25)$. If the process is continued, other peaks of concentration appear and we can see on Figure 4 , that at time $250 \mathrm{~s}$, three other peaks are present at points $\approx(0.457,0.25),(0.592,0.25),(0.698,0.25)$. These peaks of concentration remain at the same location during all the process in time. After 10 mn (Fig. 4) the peaks contain respectively $56.46 \%, 13.53 \%, 16.67 \%$ and $13.42 \%$ of the total mass of the bacteria. For each peak of concentration, the "local mass" is concentrated within one mesh element (which is the smallest part of our discretization approach) and it seems that the distribution of bacteria tends to localized Dirac masses. 


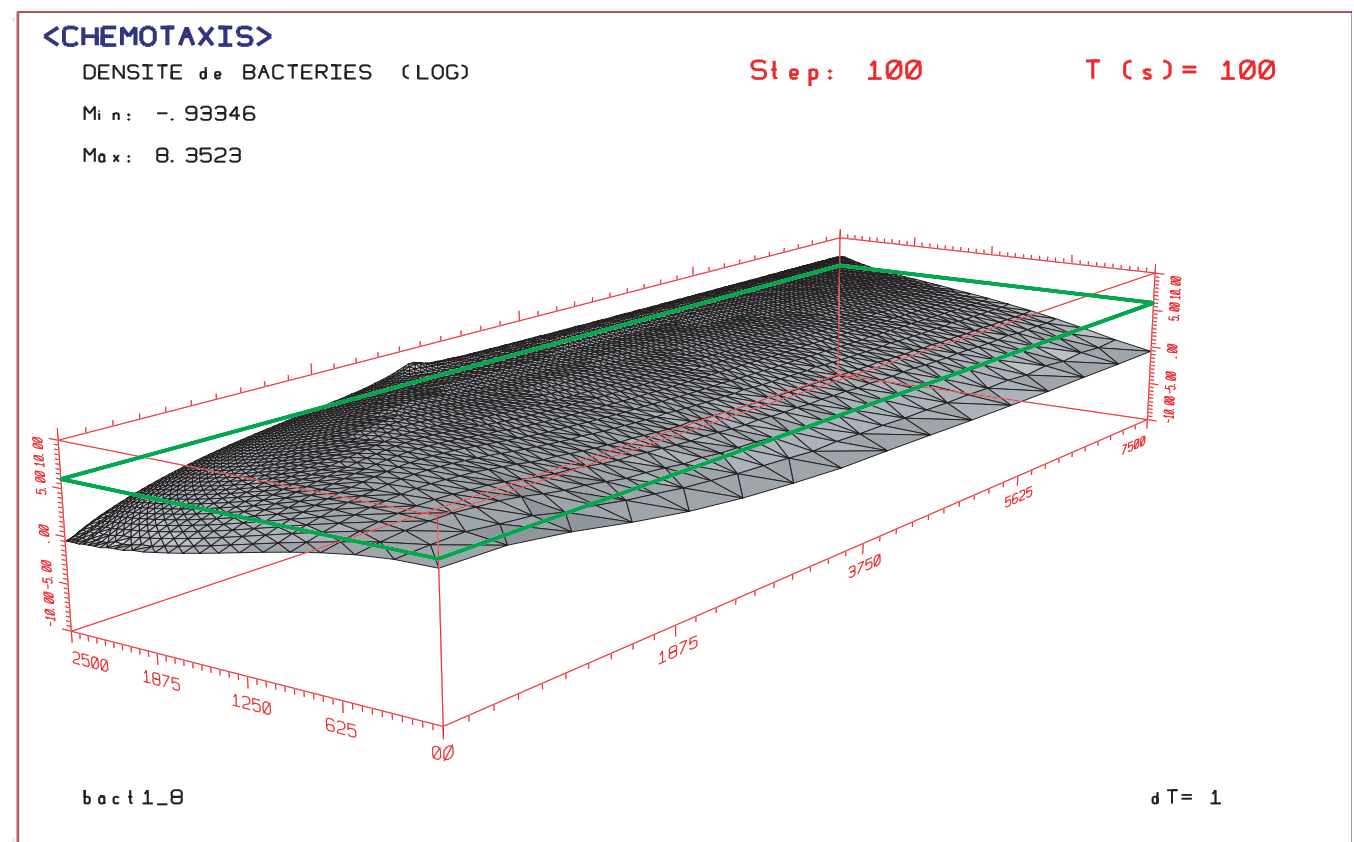

Figure 3. Bacteria concentration, (Log. scale). Attractant production model 1. Computational domain: rectangle $0.75 \mathrm{~cm} \times 0.25 \mathrm{~cm}$. Distribution after $100 \mathrm{~s}$. Initial concentration is marked, observer position near $\left(x_{\min }, y_{\min }\right)$.

\subsection{Numerical results with limited attactant production}

Figure 5 (top) presents the bacteria density distribution after $100 \mathrm{~s}$. This distribution is qualitatively quite similar to the distribution obtained with the previous model of attractant production (Fig. 3).

If the time process is continued, other peaks of concentration appear, like in the previous simulation, but the first concentration peak travels towards the center of the domain (i.e. upper right corner of the computational domain), "eating" successively all the other local peaks of bacteria concentration. Figures 5 (bottom) and 6 show the bacteria density distribution respectively at time $300 \mathrm{~s}, 600 \mathrm{~s}$ and $1000 \mathrm{~s}$. We can see that at $\mathrm{T}=1000 \mathrm{~s}$ only one peak is present (at the center of the rectangle $\Omega$ ) and that high density values are not localized over only one mesh element. This model of attractant production seems to produce localized high densities of bacteria (aggregation) which do not looks like Dirac masses. At time $\mathrm{T}=1000 \mathrm{~s}$ (Fig. 6 - bottom -), the density $\rho$ is greather or equal than $\rho_{o}$ - the initial density value - within 39 triangles, representing $0.145 \%$ of the total area of $\Omega$. These triangles contain $97.98 \%$ of the total mass of bacteria.

\section{Conclusion}

We have presented here a computational scheme which allows the simulation of chemotactic bacteria aggregation. As theoretically predicted, the density redistribution during the evolution process appears to be strongly dependent on the model used for the source (production) of attractants. With the first model (source of attractants proportional to the bacterial density $\rho$ ), several isolated peaks of bacterial concentration may appear - concentration localized over only one element, which is something like a Dirac mass at discrete level - and remain more or less at the same place along the evolution process.

Introducing a cutoff value in the production of attractants, the first peak of bacterial concentration travels to the center of the domain, "eliminating" all the secondary peaks. After a certain amount of time, high densities values remain localized on small part of the domain $(0.1$ to $1 \%)$ but not only over one mesh element. 

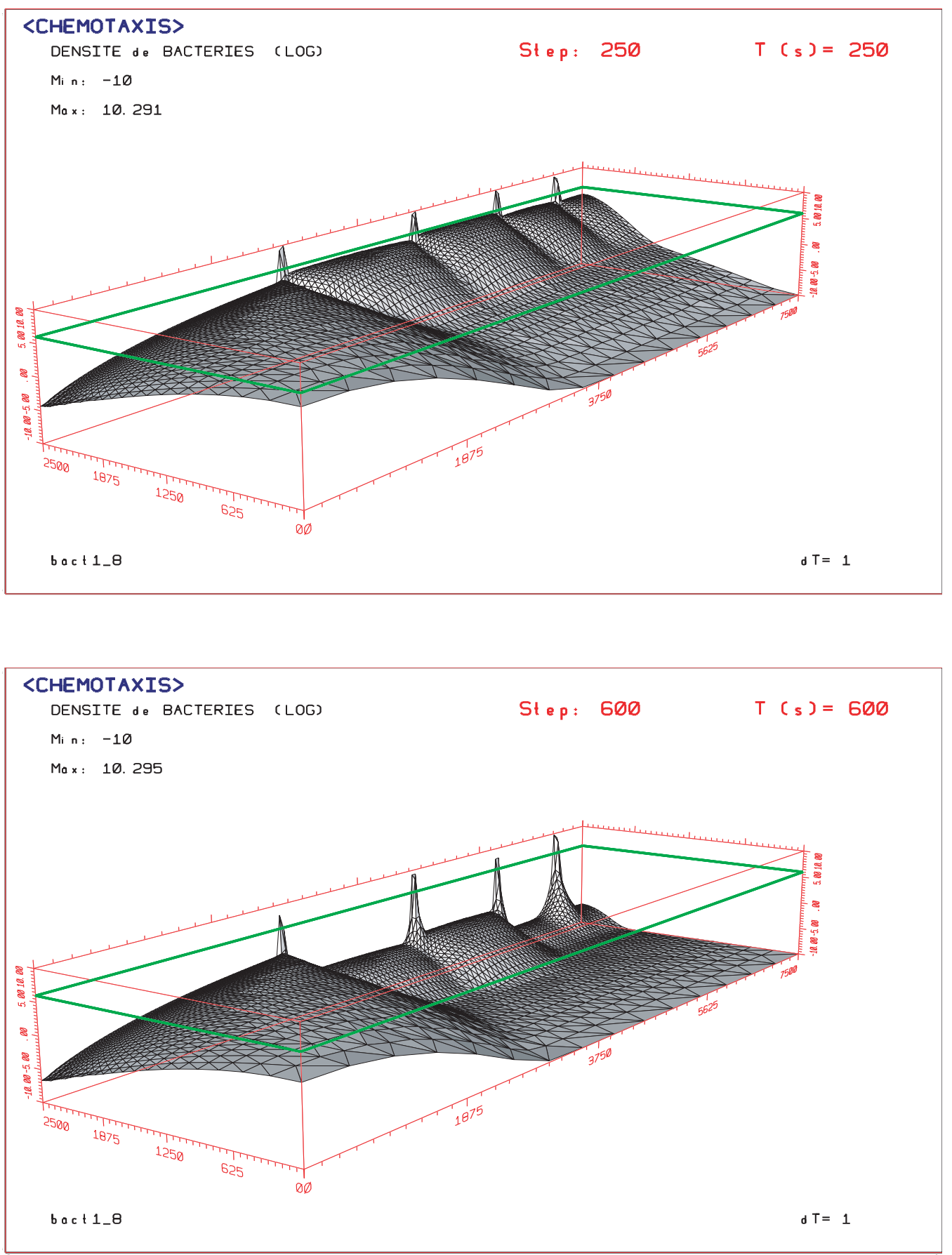

Figure 4. Bacteria concentration, (Log. scale). Attractant production model 1. Computational domain: rectangle $0.75 \mathrm{~cm} \times 0.25 \mathrm{~cm}$. Distribution after 250 and $600 \mathrm{~s}$. Initial concentration is marked, observer position near $\left(x_{\min }, y_{\mathrm{min}}\right)$. 

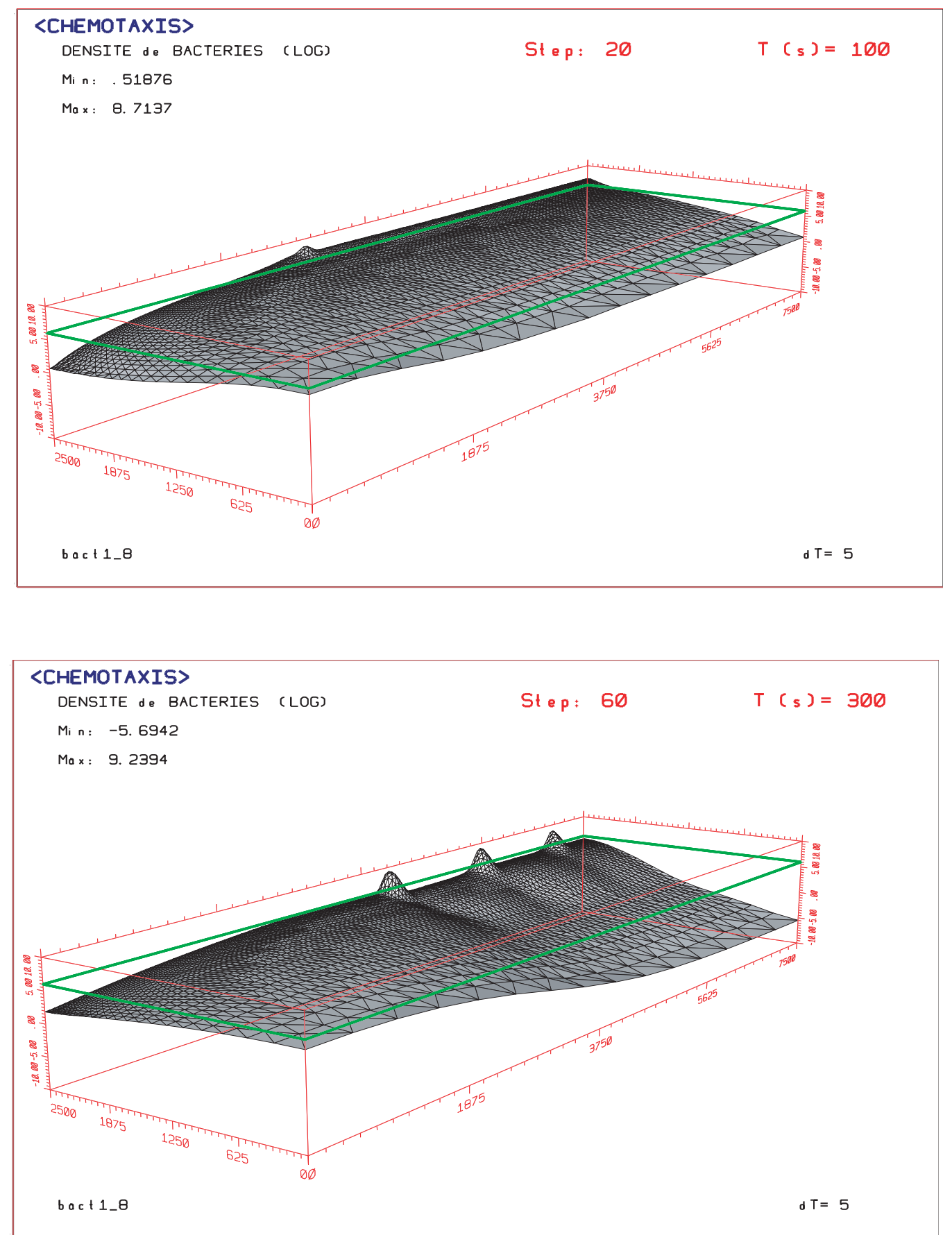

FiguRE 5. Bacteria concentration, (Log. scale). Attractant production model $2\left(\rho^{\star}=500 \rho_{0}\right)$. Computational domain: rectangle $0.75 \mathrm{~cm} \times 0.25 \mathrm{~cm}$. Observer position near $\left(x_{\min }, y_{\min }\right)$, process time: 100 and $300 \mathrm{~s}$. 

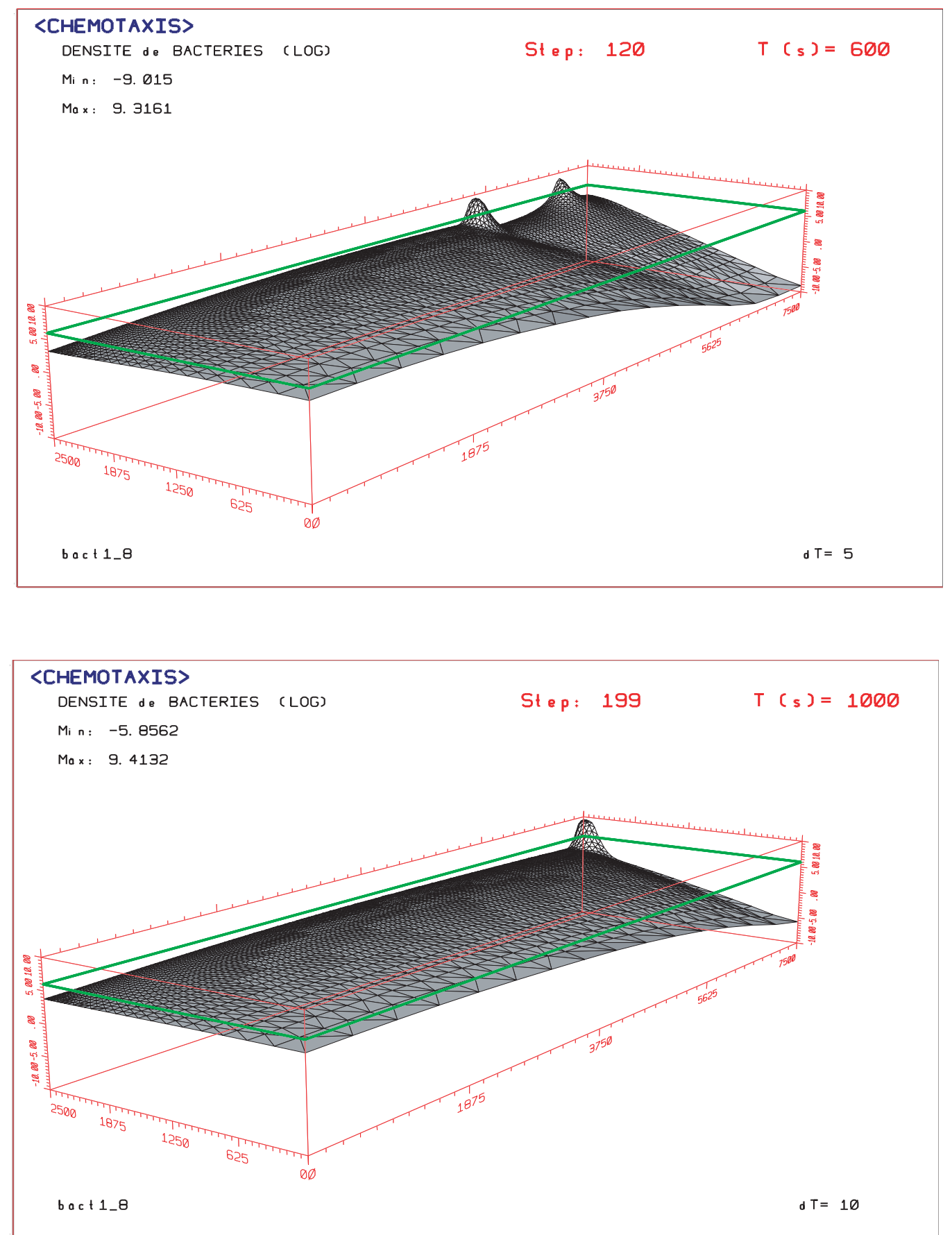

Figure 6. Bacteria concentration, (Log. scale). Attractant production model $2\left(\rho^{\star}=500 \rho_{0}\right)$. Computational domain: rectangle $0.75 \mathrm{~cm} \times 0.25 \mathrm{~cm}$. Observer position near $\left(x_{\min }, y_{\min }\right)$, process time: 600 and $1000 \mathrm{~s}$. 
Acknowledgements. Many thanks to B. Perthame for fruitful discussions and for relevant suggestions concerning this work.

\section{REFERENCES}

[1] M.D. Betterton and M.P. Brenner, Collapsing bacterial cylinders. Phys. Rev. E 64 (2001) 061904.

[2] M.P. Brenner, L.S. Levitov and E.O. Budrene, Physical mechanisms for chemotactic pattern formation bybacteria. Biophys. J. 74 (1998) 1677-1693.

[3] M.P. Brenner, P. Constantin, L.P. Kadanof, A. Schenkel and S.C. Venhataramani, Diffusion, attraction and collapse. Nonlinearity 12 (1999) 1071-1098.

[4] L. Corrias, B. Perthame and H. Zaag, A model motivated by angiogenesis. C. Rendus Acad. Sc. Paris, to appear.

[5] A El Boukili and A. Marrocco, Arclength continuation methods and applications to 2d drift-diffusion semiconductor equations. Rapport de recherche 2546, INRIA (mai 1995).

[6] A. El Boukili, Analyse mathématique et simulation numérique bidimensionnelle des dispositifs semi-conducteurs à hétérojonctions par l'approche éléments finis mixtes. Ph.D. thesis, Univ. Pierre et Marie Curie, Paris (décembre 1995).

[7] R. Glowinski and P. Le Tallec, Augmented Lagrangian and Operator Splitting Methods in Nonlinear Mechanics, Studies in Applied Mathematics. SIAM, Philadelphia (1989).

[8] M.A. Herrero and J.J.L. Velázquez, Chemotactic collapse for the keller-segel model. J. Math. Biol. 35 (1996) 177-194.

[9] M.A. Herrero, E. Medina and J.J.L. Velázquez, Finite time aggregation into a single point in a reaction-diffusion system. Nonlinearity 10 (1997) 1739-1754.

[10] F. Hecht and A. Marrocco, Numerical simulation of heterojunction structures using mixed finite elements and operator splitting, in 10th International Conference on Computing Methods in Applied Sciences and Engineering, R. Glowinski Ed., Nova Science Publishers, Le Vésinet (February 1992) 271-286.

[11] F. Hecht and A. Marrocco, Mixed finite element simulation of heterojunction structures including a boundary layer model for the quasi-fermi levels. COMPEL 13 (1994) 757-770.

[12] W. Jäger and S. Luckhaus, On explosion of solution to a system of partial differential equations modelling chemotaxis. Trans. Amer. Math. Soc. 239 (1992) 819-824.

[13] E.F. Keller and L.A. Segel, Model for chemotaxis. J. Theor. Biol. 30 (1971) 225-234.

[14] A. Marrocco and Ph. Montarnal, Simulation des modèles energy-transport à l'aide des éléments finis mixtes. C.R. Acad. Sci. Paris I 323 (1996) 535-541.

[15] Ph. Montarnal, Modèles de transport d'énergie des semi-conducteurs, études asymptotiques et résolution par des éléments finis mixtes. Ph.D. thesis, Université Paris VI (octobre 1997).

[16] A. Marrocco, 2d simulation of chemotactic bacteria aggregation. Rapport de recherche 4667, INRIA (décembre 2002).

To access this journal online:

www.edpsciences.org 